Article

\title{
Fluorine Based Superhydrophobic Coatings
}

Jean-Denis Brassard ${ }^{1,2, *}$, D.K. Sarkar ${ }^{2, *}$ and Jean Perron ${ }^{1}$

1 Anti-icing Materials International Laboratory (AMIL), Université du Québec à Chicoutimi, 555 Boulevard de 1'Université, Chicoutimi, Québec G7H 2B1, Canada; E-Mail: jean_perron@uqac.ca

2 Centre Universitaire de Recherche sur l'Aluminium (CURAL), Université du Québec à Chicoutimi, 555 Boulevard de l'Université, Chicoutimi, Québec G7H 2B1, Canada

* Authors to whom correspondence should be addressed; E-Mails: jean-denis.brassard@uqac.ca (J.D.B.); dsarkar@uqac.ca (D.K.S.); Tel.: +1-418-545-5011 (ext. 2543).

Received: 15 February 2012; in revised form: 24 April 2012 / Accepted: 2 May 2012 /

Published: 8 May 2012

\begin{abstract}
Superhydrophobic coatings, inspired by nature, are an emerging technology. These water repellent coatings can be used as solutions for corrosion, biofouling and even water and air drag reduction applications. In this work, synthesis of monodispersive silica nanoparticles of $\sim 120 \mathrm{~nm}$ diameter has been realized via Stöber process and further functionalized using fluoroalkylsilane (FAS-17) molecules to incorporate the fluorinated groups with the silica nanoparticles in an ethanolic solution. The synthesized fluorinated silica nanoparticles have been spin coated on flat aluminum alloy, silicon and glass substrates. Functionalization of silica nanoparticles with fluorinated groups has been confirmed by Fourier Transform Infrared spectroscopy (FTIR) by showing the presence of $\mathrm{C}-\mathrm{F}$ and Si-O-Si bonds. The water contact angles and surface roughness increase with the number of spin-coated thin films layers. The critical size of $\sim 119 \mathrm{~nm}$ renders aluminum surface superhydrophobic with three layers of coating using as-prepared nanoparticle suspended solution. On the other hand, seven layers are required for a 50 vol.\% diluted solution to achieve superhydrophobicity. In both the cases, water contact angles were more than $150^{\circ}$, contact angle hysteresis was less than $2^{\circ}$ having a critical roughness value of $\sim 0.700 \mu \mathrm{m}$. The fluorinated silica nanoparticle coated surfaces are also transparent and can be used as paint additives to obtain transparent coatings.
\end{abstract}


Keywords: sol-gel process; fluorinated silica nanoparticles; functionalization of nanoparticles; superhydrophobicity; fluoroalkylsilane; aluminum; water contact angle; FTIR; scanning electron microscopy; profilometry

\section{Introduction}

Superhydrophobic coatings are increasingly attractive to the industry and academia due to their unique self-cleaning properties as a result of their water repelling characteristics [1]. Fabrication of superhydrophobic coating is an inspiration from nature as water is seen to repel on many natural surfaces such as those of the lotus leaves, butterfly wings, water striders' legs, and so on [2-5]. This behavior of water drops rolling off their surfaces is due mainly to the presence of a combination of rough micro-nanostructure and low surface energy waxy materials on their surfaces. This concept has been well elaborated by Neinhuis et al. [4] on the surface of the lotus leaves which has been the classic example is the field of superhydrophobicity for researchers around the globe emphasizing the importance of the geometry and the chemistry of the surface. Inspired by this phenomenon, we have recently transformed aluminum surfaces, copper and silicon surfaces superhydrophobic by first creating surface roughness using methods such as chemical bath deposition, electrochemical methods and chemical etching methods and then modifying those surfaces by either passivating using low surface energy molecules such as stearic acid or fluoroalkyl-silane (FAS-17) or by coating with rf-sputtered Teflon thin films [6-11]. The multilayer deposition process of organic and inorganic materials [12,13] such as nanoparticles as rough hydrophobic material $[14,15]$ are also techniques to obtain superhydrophobic surfaces.

In all these cases, the surfaces exhibited water roll-off properties providing water contact angle values greater than $150^{\circ}$, which is due to the air entrapment in the gaps of the rough structure produced in the first step, resulting in a composite structure of air and solid in combination with the presence of low surface energy components on the roughened surface which reduces the affinity of water to the surface. The effect of surface roughness on superhydrophobic properties has been elucidated by Wenzel and Cassie-Baxter in their mathematical models formulated in 1940s [16,17].

Superhydrophobic surfaces find tremendous importance in the technological world where they can be applied in domains such as anti-corrosion, anti-biofouling, electrowetting and even in drag reductions [7,18-23]. However, the most important challenge faced by industries is the feasibility of large-scaling in an effective and economical manner. Therefore, in this study, we present a simple sol-gel technique where silica nanoparticles are synthesized via a Stöber process. In order to eliminate a second step of passivation or coating to obtain low surface energy, these silica nanoparticles are functionalized in the suspension using FAS-17 molecules before coating on aluminum substrates. The functionalized fluorinated silica nanoparticles suspended in a solution are then deposited via a spin-coating technique on to the aluminum substrates to obtain superhydrophobic coatings demonstrating large-scale feasibility. 


\section{Experimental Section}

The solutions containing fluorinated silica nanoparticles are prepared in the laboratory using standard Stöber process [24]. Initially, at a hotplate temperature of $50{ }^{\circ} \mathrm{C}$, ethanol and ammonium hydroxide $\left(\mathrm{NH}_{4} \mathrm{OH}\right)$ were mixed in a beaker using a magnetic stir bar. While stirring, a solution of tetraethoxysilane or TEOS $\left(\mathrm{Si}\left(\mathrm{OC}_{2} \mathrm{H}_{5}\right)_{4}\right)$ is added drop by drop in the mixed solution. The transparent mixed solution turns opaque confirming the formation of silica nanoparticles [25-27]. The synthesized silica nanoparticles are further funtionalized in an ethanolic fluoroalkylsilane or FAS17 $\left(\mathrm{C}_{16} \mathrm{H}_{19} \mathrm{~F}_{17} \mathrm{O}_{3} \mathrm{Si}\right)$ solution. The AA-6061 aluminum alloy, silicon and sodalime glass substrates were coated with the fluorinated silica nanoparticles by spin-coating processes. The nanoparticle coated films were dried at $70{ }^{\circ} \mathrm{C}$ on a hotplate to allow the ethanol and excess water to evaporate from the films. Transform Infrared spectroscopy (IRRAS) was used to evaluate the atomic bonding in the films. IRRAS (Nicolet 6700 FT-IR) is equipped with a Mid-IR MCT-A $\mathrm{N}_{2}$-cooled detector and a $\mathrm{KBr}$ beam splitter. The thin films were also analysed via a high-resolution field emission gun scanning electron microscope (FEGSEM: Hitachi SU-70) for morphological analysis. The wetting properties of the functionalized particles coated on multiple substrates were performed by measuring both static and dynamic contact angles (Krüss contact angle goniometer) at five positions on each substrate using a $5 \mu \mathrm{L}$ deionized water drop. The static contact angle has been abbreviated as CA and dynamic contact angle or contact angle hysteresis has been abbreviated as $\mathrm{CAH}$ throughout the text. The $\mathrm{CAH}$ is the difference between the advancing and the receding angle. The $\mathrm{CAH}$ measurements were made at room temperature following a very standard and commonly used experimental procedure as reported in the literature [28-30]. In this method, a water drop of volume $\sim 5 \mu \mathrm{L}$ was suspended with the needle and brought in contact with the superhydrophobic surfaces using a computer controlled device as provided by Krüss $\mathrm{GmbH}$. The contact $\mathrm{CAH}$ was measured by holding the water drop with a stationary needle in contact with the surface and moving the goniometer stage in one direction. The surface roughness of the films were measured using an optical profilometer (MicroXAM-100 HR 3D surface profilometer).

\section{Results and Discussion}

The molecular bonding of the fluorinated species as well as the silica bonds on surfaces coated with varying numbers of layers of the fluorine-functionalized silica nanoparticles were investigated with IRRAS spectral analyses. The Figure 1(A) shows the IRRAS spectra of the fluorinated silica nanoparticles spin coated aluminum substrates in one and nine layers. When one and nine layers are spin coated on the substrate, several peaks appear. The peak at $1,100 \mathrm{~cm}^{-1}$ is due to the asymmetric stretching vibration of Si-O-Si bonds in the silica nanoparticles in the thin films [31-34]. Another small peak appearing at around $800 \mathrm{~cm}^{-1}$ is associated with the bending mode of Si-O-Si bonds. Also, the peak at $480 \mathrm{~cm}^{-1}$ is associated with $\mathrm{Si}-\mathrm{O}-\mathrm{Si}$ bond for the rocking vibration [35]. This particular peak shows clearly an increase in intensity with an increase in the number of layers, proving an increase of the concentration of nanoparticles on the Al surface. Infrared analysis of the surface shows a peak around $945 \mathrm{~cm}^{-1}$ which is attributed to silicon hydroxide ( $\left.\mathrm{Si}-\mathrm{OH}\right)$ bonds present on unfunctionnalized silica nanoparticles [6]. 
Other interesting peaks are those present in the FAS17 molecules. At the beginning of the molecules, hydro-carbon bonds are present. A reasonably broad peak around $900 \mathrm{~cm}^{-1}$ is assigned to the $\mathrm{C}-\mathrm{H}$ bonds. Continuing on the molecules, fluoro-carbon bonds are present. The existence of C-F bonds in the form of $\mathrm{CF}, \mathrm{CF}_{2}$ or $\mathrm{CF}_{3}$ are also located at $610,730,960$ and $1,250 \mathrm{~cm}^{-1}[33,34,36,37]$. The most important peak appears at $1,145 \mathrm{~cm}^{-1}$. This particular peak represents a Si-O-C bond which confirms that the FAS17 molecule is clearly attached to silica nanoparticles. The presence of additional new peaks arising from the fluorinated functional groups present in the films confirms that the nanoparticles of silica are functionalized by fluorine from the FAS17 molecules. Figure 1(B) shows on the top a representation of a silica nanoparticle covered with a thin film of FAS17. On the bottom of the figure, a molecular representation of the fluorinated nanoparticles is presented with their corresponding wavenumbers.

Figure 1. (A) Transform Infrared spectroscopy (IRRAS) spectra of fluorinated silica nanoparticles coated aluminum surfaces as a function of the number of layers. (B) Schematic presentation of a silica nanoparticle functionalized with a thin layer of FAS17 (top) presented along with the corresponding wavenumbers on the IR spectra (bottom).

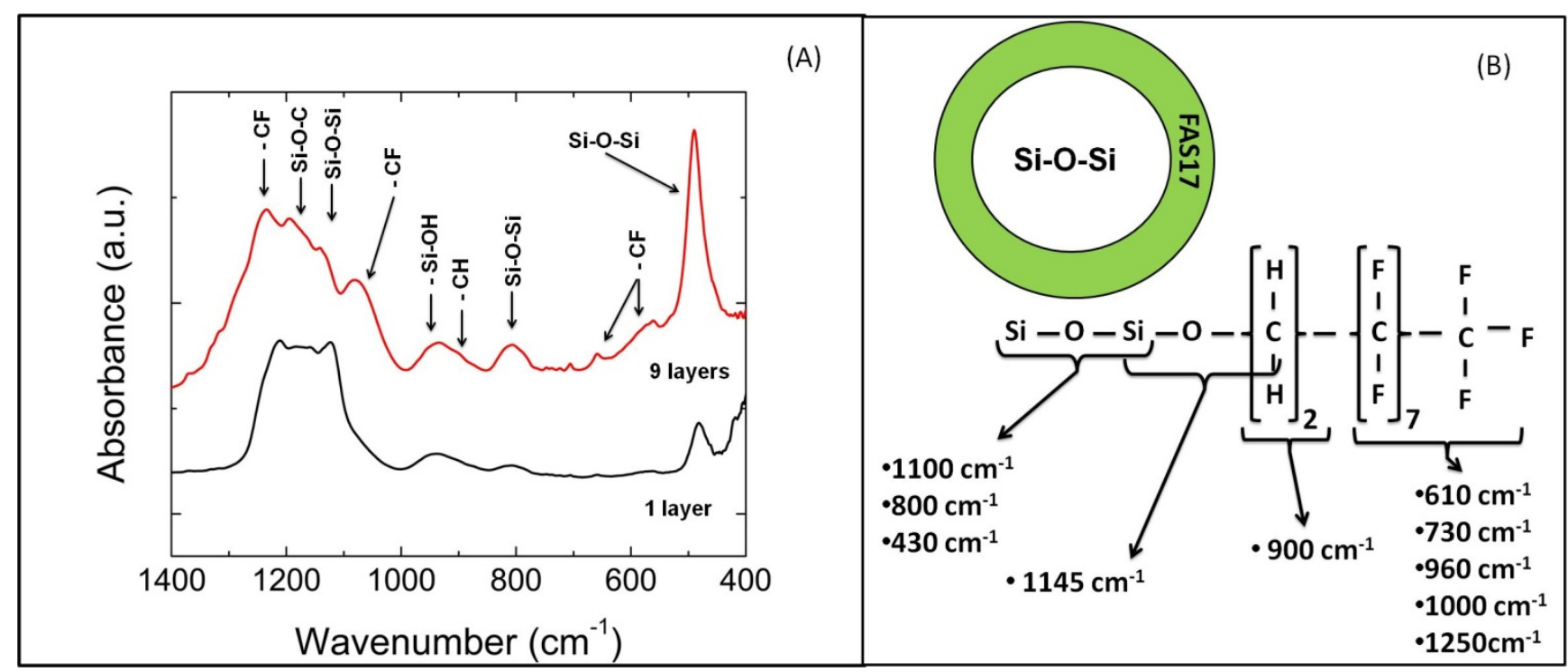

We have previously reported that that the size of silica nanoparticles can be easily controlled through the concentration of catalyst [6,24,38,39]. Figure 2 shows SEM images of spherically shaped fluorinated silica nanoparticles of diameter $119 \pm 12 \mathrm{~nm}$ deposited in three layers on aluminum substrates. The inset of Figure 2(A) shows the image of a water drop placed on this aluminum substrate surface showing a very high water contact angle of $\sim 152^{\circ}$. It is clear from these images that this surface is composed of several micrometer sized clusters of these spherical nanoparticles presenting randomly distributed holes and cracks by which the clusters are separated. It can also be seen from the higher magnification image in Figure 2(B) that these spherical fluorinated silica nanoparticles are also randomly stacked in steps of layers presenting different depths. Such an arrangement of randomly stacked spherical nanoparticles in clusters separated by cracks and holes provides the surface a rough micro-nanopattern. Nozawa et al. [38] obtained $110 \pm 20 \mathrm{~nm}$ silica 
nanoparticles using the Stöber process at a $\mathrm{NH}_{4} \mathrm{OH} / \mathrm{TEOS}$ molar ratio of 10, however, in our case, the size of the nanoparticles obtained under the same conditions are $\sim 10 \mathrm{~nm}$ larger $(119 \pm 12 \mathrm{~nm})$. The larger size in our case is attributed to the presence of an outer shell of fluorinated silane bonded around the silica nanospheres as a result of their functionalization using FAS-17 molecules as presented schematically in Figure 1(B).

Figure 2. (A) SEM images of fluorinated silica nanoparticles of $119 \pm 12 \mathrm{~nm}$ diameter deposited on aluminum substrates in three layers by spin coating; Inset of Figure 2 (A) shows the image of a water drop placed on this surface exhibiting a very high water contact angle of $\sim 152^{\circ}$, and (B) higher magnification image of (A).

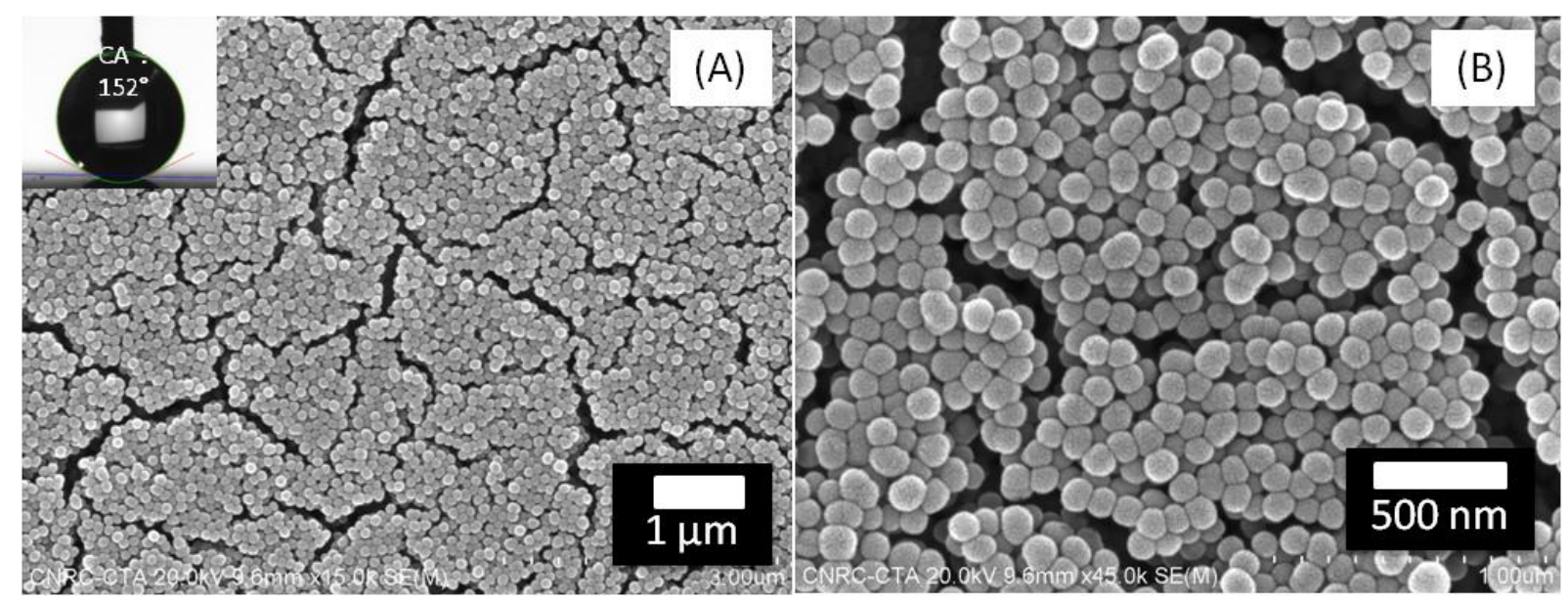

Water contact angle measurements were used to evaluate the wetting properties of the prepared surfaces. Increased contact angles are, partially, increased because of roughness, which increased air entrapment as stated by Cassie-Baxter equation. Figure 3 (A) shows the contact angles variation on the aluminum surfaces coated using the as-prepared fluorinated silica nanoparticles in the solution as well as the ones coated by diluting the same. In both cases, the water contact angle values were found to increase with the increase in the number of layers of the fluorinated silica nanoparticles deposited on the substrates until a critical number of layers was reached after which the water contact angle values remained similar on the surfaces coated using as-prepared solution. However, higher water contact angle values were obtained on surfaces coated using the as-prepared fluorinated silica nanoparticles solution. For example, on the aluminum surfaces coated with one layer of as-prepared solution, an increased water contact angle value of $137 \pm 1^{\circ}$ was obtained, making these surfaces hydrophobic since a clean aluminum surface provides a water contact angle of only $\sim 70^{\circ}$. The surface was rendered superhydrophobic when the number of layers was increased to three providing a higher water contact angle value of $152 \pm 1^{\circ}$. This increase in water contact value is attributed to the rough micro-nanomorphogical pattern obtained on these surfaces (Figure 2) as well as the presence of low surface energy fluorinated species present on these surfaces as confirmed by the IRRAS analyses (Figure 1(A)). This emphasizes the importance of the presence of both surface roughness as well as low surface energy, a phenomenon reported on lotus effect [4]. Further, the influence of surface roughness in leading to a large amount of air entrapment in the gaps of the rough structure to result in a composite structure of air and solid has been well elucidated by Cassie and Baxter [16]. With a further 
increase in the number of layers to five, the water contact angle values slightly increased to $\sim 153^{\circ}$ and remained similar when coated with seven and nine layers. Therefore, three layers of coating may be considered as a critical parameter for obtaining superhydrophobic properties when the coating is made with the as-prepared solution. Similarly, an increasing tendency in water contact angle values was observed also in cases of coatings prepared with the dilute solution. However, the water contact angle reached lower values when the same number of layers was used as with the as-prepared solution. Such lower values may be attributed to the lower concentration of the fluorinated silica nanoparticles in the diluted solution, possibly resulting in morphologies with less surface roughness (Figure 3(B)). However, with the diluted solution, superhydrophobic properties were indeed obtained when the numbers of layers deposited on the surface was increased. For example, with only one layer coated with the dilute solution, the water contact angle increased to $128 \pm 2^{\circ}$ (although this is lower than on the same coating using as-prepared solution). With three layers, the contact angle reached $135 \pm 3^{\circ}$ which further increased to $144 \pm 1^{\circ}$ on deposition of five layers. With seven and nine layers of coating, the contact angle values reached to a high of $150 \pm 1^{\circ}$ and $151 \pm 1^{\circ}$, respectively. These values show that a critical number of seven layers was required to attain superhydrophobicity when using dilute solution as compared to only three layers that was sufficient to obtain this property with the as-prepared solution of the fluorinated silica nanoparticles.

Although superhydrophobic properties could be obtained using both as-prepared and diluted solutions, the difference in the critical number of layers required in obtain these properties is attributed to the difference in the surface roughness obtained on the corresponding surfaces. Figure 3(B) shows the variation in the root mean square (rms) roughness measured on the surfaces prepared with varying numbers of layers coated using the two different solutions deposited on aluminum substrates. As an example, a three dimensional surface profile obtained during the rms measurements of aluminum surface prepared by coating three layers using as prepared solution has been presented in the inset of Figure 3(B). These graphs show that the contact angle and the roughness vary proportionally as it is clear that the water contact angle increases with increase in surface roughness until a critical roughness value is reached. Figure 3(C) shows a plot of water contact angle as a function of rms roughness on the coatings prepared using as prepared solution. We have previously reported a detailed mathematical model to show the relationship between the roughness and water contact angle in reference to Cassie model [6]. Huang et al. [40] have also shown similar behavior on their electrochemically deposited copper state surface. On the coatings prepared using the as-prepared solution, an rms value of $0.60 \pm 0.10 \mu \mathrm{m}$ on a one layer coating increased to $0.69 \pm 0.01 \mu \mathrm{m}$ when coated with three layers where superhydrophobic properties were obtained with a contact angle above $150^{\circ}$. With further increase in the number of layers to five, seven and nine, the rms roughness remained unchanged $(\sim 0.71 \mu \mathrm{m})$. The similar rms values on the coatings prepared with more than three layers shows that increasing the number of layers further does not cause any change in the surface roughness and therefore provides the same contact angle values on these surfaces. Therefore, an rms roughness of $0.69 \pm 0.01 \mu \mathrm{m}$ obtained with three layer coating is found to be a critical value in obtaining superhydrophobic properties. Similarly, in case of the coatings prepared using diluted solution, the rms values show that superhydrophobic properties were only reached when the rms values reached $0.70 \pm 0.02 \mu \mathrm{m}$ when coated with seven layers on the substrate providing a water contact angle value of $150 \pm 1^{\circ}$. These values were lower on the surfaces coated using less layers, that is, $0.57 \pm 0.10 \mu \mathrm{m}$, 
$0.61 \pm 0.03 \mu \mathrm{m}$ and $0.67 \pm 0.03 \mu \mathrm{m}$, respectively on the surfaces coated with one, three and five layers, which provided water contact angles lower than $150^{\circ}$. With nine layers of coating, the rms value slightly increased to $0.72 \pm 0.05 \mu \mathrm{m}$, maintaining superhydrophobic properties. Therefore, a critical rms roughness value of $0.69 \pm 0.01 \mu \mathrm{m}$ in case of as-prepared solution which is not very different from the rms values of $0.70 \pm 0.02 \mu \mathrm{m}$ in case of diluted solution is required for obtaining superhydrophobic properties, however, the presence of low surface energy fluorinated components is another key factor in obtaining these properties. In our case, the presence of a low surface energy fluorinated component is inherent as the silica nanoparticles are funtionalized with FAS-17 molecules prior to deposition on the substrate surfaces. The combination of the critical surface roughness of $\sim 0.70 \mu \mathrm{m}$ and presence of fluorinated species as confirmed by IRRAS analyses leads to the superhydrophobic properties with water roll-off properties.

Figure 3. (A) Water contact angle variation, and (B) rms roughness as a function of number of layers coated using as-prepared (black) and dilute solution (red); (C) relationship between water contact angle and rms roughness as obtained on the coatings prepared using as-prepared solution on aluminum substrates; and (D) water contact angle variation with number of layers coated using dilute solution on different substrates, namely, aluminum (black), silicon (100) (red) and sodalime glass (blue). Inset shows transparency of our superhydrophobic coatings on glass substrates.

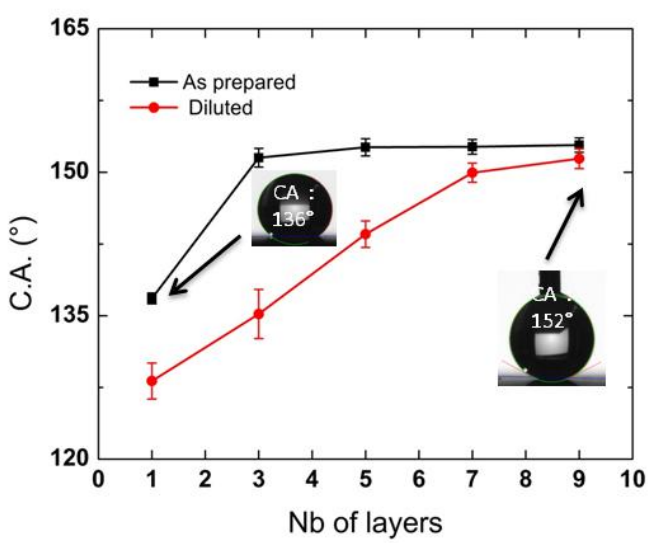

(A)

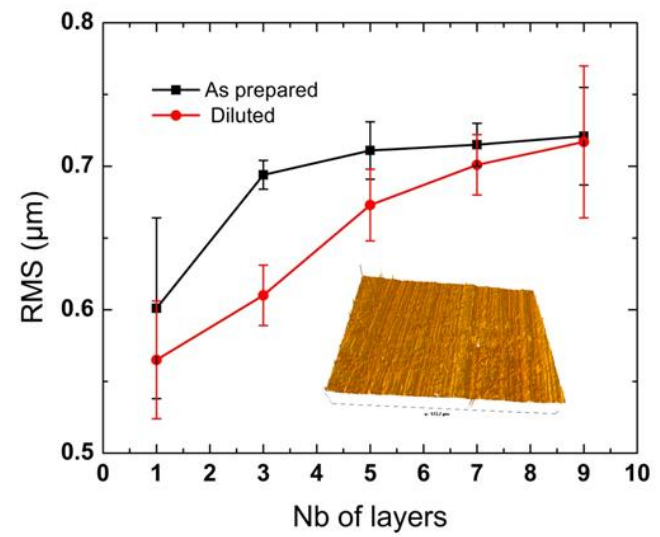

(B)

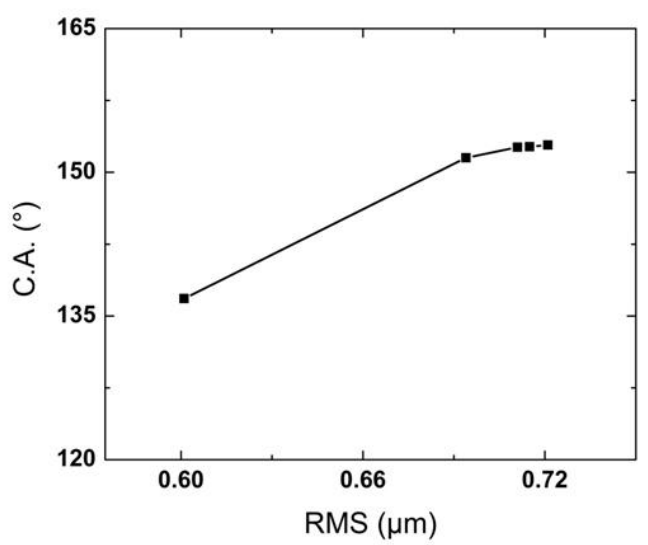

(C)

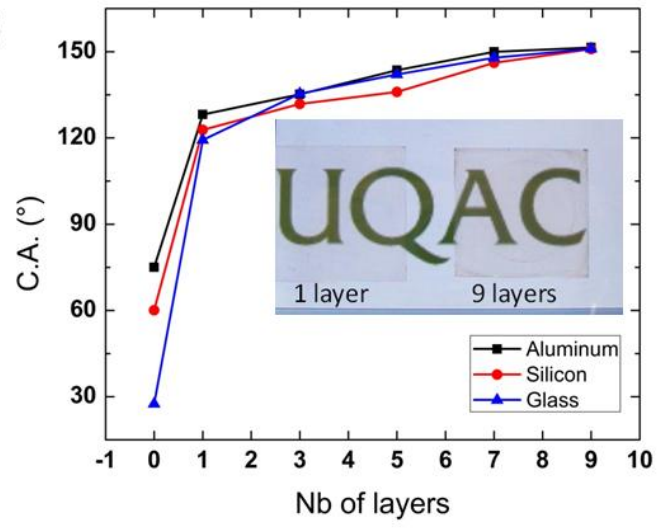

(D) 
The uniformity of the coating has been verified on various substrates as shown in Figure 3(D). In addition to aluminum substrates, the feasibility of the coatings were also tested on silicon (100) and sodalime glass substrates. Figure 3 (D) shows the water contact angle behavior for all the three substrates coated with diluted solution of fluorinated silica nanoparticles. The water contact values obtained on as-received flat substrates of $\operatorname{Si}(100)$ and glass are $\sim 60^{\circ}$ and $\sim 30^{\circ}$, respectively. In both cases, the water contact angle value increases with an increase in number of deposited layers. With just one layer of coating, the surface reaches the hydrophobic zone by providing water contact values greater than $90^{\circ}$ in case of all the three substrates. Superhydrophobic properties are reached by increasing the number of deposited layers. The feasibility of obtaining superhydrophobicity on different substrate surfaces can have great potential in industrial use. As a potential for applications on glass windows or car wind-shields, we have provided images of glass substrates coated with one layer and nine layers of diluted solution in the inset of Figure 3(B). It is clear from these images that one layer or nine layers does not dramatically alter the transparency of the glass following coating visually, the only difference being that the one layer coating is hydrophobic while the nine layer coating is superhydrophobic. Such coatings also present promise for applications for aesthetic purposes when coated on opaque substrates.

Figure 4. Contact angle hysteresis $(\mathrm{CAH})$ as a function of number of layers coated using as-prepared (black) and diluted solution (red). Inset image at the left shows high CAH of $\sim 15^{\circ}$ for one layer coating and right shows low CAH of $2^{\circ}$ for nine layers coating.

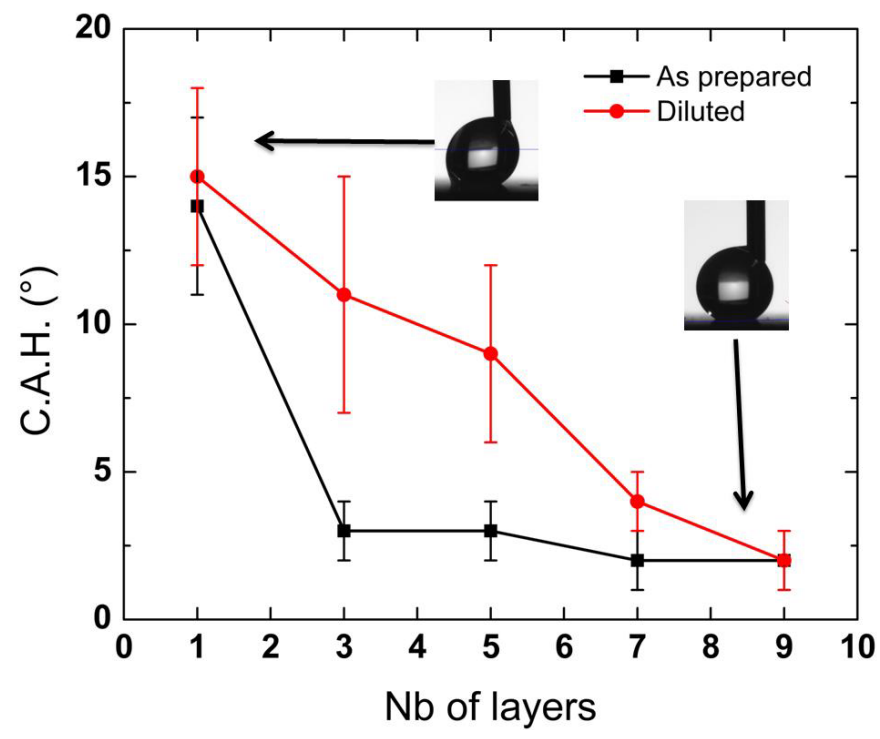

The combined effect of CA and CAH determine the self-cleaning properties [29]. A surface having $\mathrm{CA}$ more than $150^{\circ}$ and $\mathrm{CAH}$ less than 5 degree shows self-cleaning properties. On the other hand, a surface showing $\mathrm{CA}$ more than $150^{\circ}$ and $\mathrm{CAH}$ more than $10^{\circ}$ does not show the self-cleaning properties [41]. In the view of claiming the self-cleaning properties of our superhydrophobic surfaces, we have performed both $\mathrm{CA}$ and $\mathrm{CAH}$ experiments. The $\mathrm{CA}$ results have been discussed in the previous section. In this section we describe the results of dynamic contact or CAH. Figure 4 shows the $\mathrm{CAH}$ data of the coatings prepared with fluorinated silica nanoparticles on flat aluminum substrates. One layer coating using both as-prepared as well as diluted solution provides CAH of 
$14 \pm 3^{\circ}$ and $15 \pm 3^{\circ}$, respectively. The $\mathrm{CAH}$ reduced to $3 \pm 1^{\circ}$ with three layers coatings of as-prepared solution. However, in the three layer coatings using diluted solution, the CAH was $11 \pm 4^{\circ}$. The CAH reduced to $2 \pm 1^{\circ}$ for the coatings obtained by seven layers using as-prepared solution. On the other hand, the seven layer coatings with the diluted solution provided a CAH of $4 \pm 1^{\circ}$. Finally, for both the solutions with nine layers of coatings, the $\mathrm{CAH}$ reduced to $2 \pm 1^{\circ}$. Therefore, the self-cleaning properties (coating having CA more than $150^{\circ}$ and $\mathrm{CAH}$ less than $5^{\circ}$ ) can be obtained with three layer coatings using as-prepared solution. However, for diluted solution a coating with seven layers is required.

The mechanical properties of the superhydrophobic coatings are very important for their uses against surface erosion, friction as well as corrosion protection [42-44]. Keeping these important applications in mind, we will be investigating the mechanical properties of our superhydrophobic coatings in future.

\section{Conclusions}

Solutions of monodispersive spherical fluorinated silica nanoparticles of $\sim 120 \mathrm{~nm}$ have been prepared by sol-gel processes and have been used to prepare thin films on flat aluminum, silicon and glass substrates by spin-coating processes. FAS-17 molecules are used for the fluorination of the silica nanoparticles. IRRAS spectra show the presence of $\mathrm{C}-\mathrm{F}$ and $\mathrm{Si}-\mathrm{O}$ bonds resulting from the functionalization of silica nanoparticles by FAS-17 molecules. An increase in the surface area of the $\mathrm{C}-\mathrm{F}$ and Si-O peaks has been encountered in the spectra with an increase in the number of layers used for coating. The water contact angle values were found to increase with increasing numbers of layers until a critical number of layers: three in the case of as-prepared solution used for coating and seven in case of the diluted solution used for coating. As we have achieved the contact angle of more than $150^{\circ}$ and $\mathrm{CAH}$ less than $2^{\circ}$, we consider our coatings possess self-cleaning properties. Water contact angle in relation to surface roughness measurements show both parameters are proportional as water contact angle increases with an increase in surface roughness. The roughness measurements also show that the rms values increase with the increase in the number of layers coated. When applied on other substrates, like silicon or glass, superhydrophobicity is obtained by tuning the number of layers of deposition. The transparency of the coating has been demonstrated on glass substrates, which shows the great potential of such coatings in industrial use on large scale, such as on windows and car windshields as well as for aesthetic purposes on opaque substrates. With its good optical properties, these fluorinated silica nanoparticles can be used as paint additives to obtain large-scale superhydrophobic coatings.

\section{Acknowledgments}

The authors would like to thank the Natural Sciences and Engineering Research Council of Canada (NSERC) for the financial support. The authors would also thank D. Kocaefe for access of the profilometry apparatus at CURAL research centre, H. Gregoire, at NRC-ATC, Chicoutimi for the SEM analysis and N. Saleema at NRC-ATC, Chicoutimi for IR analysis as well as the critical reading of the manuscript. 


\section{Conflict of Interest}

The authors declare no conflict of interest.

\section{References}

1. Liu, X.; Liang, Y.; Zhou, F.; Liu, W. Extreme wettability and tunable adhesion: Biomimicking beyond nature? Soft Matter 2012, 8, 2070-2086.

2. Gao, X.; Jiang, L. Water-repellent legs of water striders. Nature 2004, 432, doi: 10.1038/432036a.

3. Huang, J.; Wang, X.; Wang, Z.L. Controlled replication of butterfly wings for achieving tunable photonic properties. Nano Lett. 2006, 6, 2325-2331.

4. Neinhuis, C.; Barthlott, W. Characterization and distribution of water-repellent, self-cleaning plant surfaces. Ann. Botan. 1997, 79, 667-677.

5. Zheng, Y.; Gao, X.; Jiang, L. Directional adhesion of superhydrophobic butterfly wings. Soft Matter 2007, 3, 178-182.

6. Brassard, J.-D.; Sarkar, D.K.; Perron, J. Synthesis of monodisperse fluorinated silica nanoparticles and their superhydrophobic thin films. ACS Appl. Mater. Interfaces 2011, 3, 3583-3588.

7. Huang, Y.; Sarkar, D.K.; Chen, X.G. A one-step process to engineer superhydrophobic copper surfaces. Mater. Lett. 2010, 64, 2722-2724.

8. Safaee, A.; Sarkar, D.K.; Farzaneh, M. Superhydrophobic properties of silver-coated films on copper surface bye galvanic exchange reaction. Appl. Surf. Sci. 2008, 254, 2493-2498.

9. Saleema, N.; Sarkar, D.K.; Gallant, D.; Paynter, R.W.; Chen, X.G. Chemical nature of superhydrophobic aluminum alloy surfaces produced via a one-step process using fluoroalkyl-silane in a base medium. ACS Appl. Mater. Interfaces 2011, 3, 4775-4781.

10. Sarkar, D.K.; Farzaneh, M.; Paynter, R.W. Superhydrophobic properties of ultrathin rf-sputtered Teflon films coated etched aluminum surfaces. Mater. Lett. 2008, 62, 1226-1229.

11. Sarkar, D.K.; Saleema, N. One-step fabrication process of superhydrophobic green coatings. Surf.Coat. Technol. 2010, 204, 2483-2486.

12. Garcia, N.; Benito, E.; Tiemblo, P.; Hasan, M.M.B.; Synytska, A.; Stamm, M. Chemically guided topography in alkylsilane- and oligosiloxane-modified silica nanoparticle coatings: From very hydrophobic surfaces to "pearl” bouncing droplets. Soft Matter 2010, 6, 4768-4776.

13. Taurino, R.; Fabbri, E.; Messori, M.; Pilati, F.; Pospiech, D.; Synytska, A. Facile preparation of superhydrophobic coatings by sol-gel processes. J. Colloid Interface Sci. 2008, 325, 149-156.

14. Liu, H.; Szunerits, S.; Pisarek, M.; Xu, W.; Boukherroub, R. Preparation of superhydrophobic coatings on zinc, silicon, and steel by a solution-immersion technique. ACS Appl. Mater. Interfaces 2009, 1, 2086-2091.

15. Liu, H.; Szunerits, S.; Xu, W.; Boukherroub, R. Preparation of superhydrophobic coatings on zinc as effective corrosion barriers. ACS Appl. Mater. Interfaces 2009, 1, 1150-1153.

16. Cassie, A.B.D.; Baxter, S. Wettability of porous surfaces. Trans. Faraday Soc. 1944, 40, 546-551.

17. Wenzel, R.N. Resistance of solid surfaces to wetting by water. Ind. Eng. Chem. 1936, 28, 988-994.

18. Bushnell, D.M.; Moore, K.J. Drag reduction in nature. Annu. Rev. Fluid Mech. 1991, 23, 65-79. 
19. Cao, L.; Jones, A.K.; Sikka, V.K.; Wu, J.; Gao, D. Anti-icing superhydrophobic coatings. Langmuir 2009, 25, 12444-12448.

20. Carlborg, C.F.; van der Wijngaart, W. Sustained superhydrophobic friction reduction at high liquid pressures and large flows. Langmuir 2010, 27, 487-493.

21. Carré, A.; Mittal, K.L. Superhydrophobic Surfaces; VSP: Leiden, The Netherlands, 2009.

22. Karmouch, R.; Ross, G.G. Superhydrophobic wind turbine blade surfaces obtained by a simple deposition of silica nanoparticles embedded in epoxy. Appl. Surf. Sci. 2010, 257, 665-669.

23. Verplanck, N.; Coffinier, Y.; Thomy, V.; Boukherroub, R. Wettability switching techniques on superhydrophobic surfaces. Nanoscale Res. Lett. 2007, 2, 577-596.

24. Stöber, W.; Fink, A.; Bohn, E. Controlled growth of monodisperse silica spheres in the micron size range. J. Colloid Interface Sci. 1968, 26, 62-69.

25. Chen, S.-L.; Dong, P.; Yang, G.-H.; Yang, J.-J. Kinetics of formation of monodisperse colloidal silica particles through the hydrolysis and condensation of tetraethylorthosilicate. Ind. Eng. Chem. Res. 1996, 35, 4487-4493.

26. Yang, H.; Pi, P.; Cai, Z.-Q.; Wen, X.; Wang, X.; Cheng, J.; Yang, Z.-R. Facile preparation of super-hydrophobic and super-oleophilic silica film on stainless steel mesh via sol-gel process. Appl. Surf. Sci. 2010, 256, 4095-4102.

27. Bravo, J.; Zhai, L.; Wu, Z.; Cohen, R.E.; Rubner, M.F. Transparent superhydrophobic films based on silica nanoparticles. Langmuir 2007, 23, 7293-7298.

28. Callies, M.; Chen, Y.; Marty, F.; Pépin, A.; Quéré, D. Microfabricated textured surfaces for super-hydrophobicity investigations. Microelectron. Eng. 2005, 78-79, 100-105.

29. Quéré, D. Non-sticking drops. Reports on Progress in Physics 2005, 68, 2495-2532.

30. Sarkar, D.K.; Farzaneh, M.; Paynter, R.W. Superhydrophobic properties of ultrathin rf-sputtered Teflon films coated etched aluminum surfaces. Mater. Lett. 2008, 62, 1226-1229.

31. Hozumi, A.; Takai, O. Preparation of ultra water-repellent films by microwave plasma-enhanced CVD. Thin Solid Films 1997, 303, 222-225.

32. Latthe, S.S.; Imai, H.; Ganesan, V.; Rao, A.V. Superhydrophobic silica films by sol-gel co-precursor method. Appl. Surf. Sci. 2009, 256, 217-222.

33. Teshima, K.; Sugimura, H.; Inoue, Y.; Takai, O. Gas barrier performance of surface-modified silica films with grafted organosilane molecules. Langmuir 2003, 19, 8331-8334.

34. Zhao, Y.; Li, M.; Lu, Q.; Shi, Z. Superhydrophobic polyimide films with a hierarchical topography: Combined replica molding and layer-by-layer assembly. Langmuir 2008, 24, 12651-12657.

35. Sarkar, D.K.; Brassard, D.; Khakani, M.A.E.; Ouellet, L. Dielectric properties of sol-gel derived high-k titanium silicate thin films. Thin Solid Films 2007, 515, 4788-4793.

36. Hozumi, A.; Takai, O. Effect of hydrolysis groups in fluoro-alkyl silanes on water repellency of transparent two-layer hard-coatings. App. Surf. Sci. 1996, 103, 431-441.

37. Latthe, S.S.; Imai, H.; Ganesan, V.; Rao, A.V. Superhydrophobic silica films by sol-gel co-precursor method. Appl. Surf. Sci. 2009, 256, 217-222.

38. Nozawa, K.; Gailhanou, H.; Raison, L.; Panizza, P.; Ushiki, H.; Sellier, E.; Delville, J.P.; Delville, M.H. Smart control of monodisperse stöber silica particles: Effect of reactant addition rate on growth process. Langmuir 2004, 21, 1516-1523. 
39. Qu, A.L.; Wen, X.F.; Pi, P.H.; Cheng, J.; Yang, Z.R. Study on superhydrophobicity of composite silica film surface. Wuji Cailiao Xuebao/J. Inorg. Mater. 2008, 23, 373-378.

40. Huang, Y.; Sarkar, D.K.; Chen, X.G. Fabrication of superhydrophobic surfaces on aluminum alloy via electrodeposition of copper followed by electrochemical modification. Nano Micro Lett. 2011, 3, 160-165.

41. Dufour, R.; Harnois, M.; Coffinier, Y.; Thomy, V.; Boukherroub, R.; Senez, V. Engineering sticky superomniphobic surfaces on transparent and flexible PDMS substrate. Langmuir 2010, 26, $17242-17247$.

42. Ke, Q.; Fu, W.; Jin, H.; Zhang, L.; Tang, T.; Zhang, J. Fabrication of mechanically robust superhydrophobic surfaces based on silica micro-nanoparticles and polydimethylsiloxane. Surf. Coat. Technol. 2011, 205, 4910-4914.

43. Lakshmi, R.V.; Bharathidasan, T.; Basu, B.J. Superhydrophobic sol-gel nanocomposite coatings with enhanced hardness. Appl. Surf. Sci. 2011, 257, 10421-10426.

44. Ou, J.; Liu, M.; Li, W.; Wang, F.; Xue, M.; Li, C. Corrosion behavior of superhydrophobic surfaces of Ti alloys in $\mathrm{NaCl}$ solutions. Appl. Surf. Sci. 2012, 258, 4724-4728.

(C) 2012 by the authors; licensee MDPI, Basel, Switzerland. This article is an open access article distributed under the terms and conditions of the Creative Commons Attribution license (http://creativecommons.org/licenses/by/3.0/). 